\title{
Childhood psychosocial adversity and adult cortisol patterns
}

\author{
Chris Power, Claudia Thomas, Leah Li and Clyde Hertzman
}

\section{Background}

Cortisol levels may be altered in childhood in association with maltreatment (neglect, abuse and witnessing abuse) and other adversities, yet little is known about whether effects on cortisol persist into later life.

\section{Aims \\ To establish whether childhood psychosocial adversities predict cortisol levels in mid-adulthood.}

\section{Method}

Childhood psychosocial adversities were ascertained in the 1958 British birth cohort and cortisol was measured in two saliva samples, one $45 \mathrm{~min}$ after awaking $\left(T_{1}\right)$ and the other $3 \mathrm{~h}$ later the same day $\left(T_{2}\right)$, from 6524 participants aged 45 years.

\section{Results}

No association was seen for abuse or household dysfunction in childhood and adult cortisol levels. In women but not men, $T_{1}$ cortisol was lowered by $7.9 \%$ per unit increase in childhood neglect score (range $0-3$ ); $T_{1}$ to $T_{2}$ cortisol decline was less steep. High levels of maltreatment (abuse, neglect, witnessed abuse) were associated with $>25 \%$ lower $T_{1}$ cortisol in both men and women, and $24 \%$ higher $T_{2}$ cortisol for men after adjustment for concurrent depressive/anxiety symptoms.

\section{Conclusions}

In a non-clinical population, cumulative maltreatments in childhood were associated with flattened morning cortisol secretion in mid-adult life.

\section{Declaration of interest}

None.
Cortisol levels may be altered in childhood in association with maltreatment (neglect, abuse and witnessing abuse) and other adversities, ${ }^{1-4}$ yet, with few longitudinal studies, ${ }^{5}$ little is known about whether effects on cortisol persist into later life. An extensive literature demonstrates the increased risk of adult psychological disorder among those maltreated in childhood. ${ }^{6,7}$ Childhood experiences may have a lifelong influence on the function of the hypothalamic-pituitary-adrenal (HPA) axis and thus on the regulation of cortisol. ${ }^{4}$ It has also been suggested that childhood maltreatment may sensitise the HPA axis resulting in dysfunction and vulnerability to psychological disorders such as depression. ${ }^{8}$ Such hypotheses beg the question of whether childhood adversity has a persisting association with cortisol in mid-life. Using data from the 1958 British birth cohort, we aimed to establish whether co-occurring childhood adversities such as maltreatment and household dysfunction were related to cortisol patterns in mid-adulthood (age 45 years) in the general population. A second objective was to establish whether adult anxiety and depression mediate or moderate any association between childhood adversity and cortisol secretion.

\section{Method}

\section{Study population}

The 1958 cohort comprised a population of about 17000 live births in England, Scotland and Wales, all born in one week in March 1958 and followed up at ages 7, 11, 16, 23, 33 and 42 years. ${ }^{9}$ At age 44-45 years, i.e. during 2002-2004, a biomedical survey was undertaken comprising a home interview by a trained research nurse, two short self-completed questionnaires, physical measurements, blood samples and a saliva sample obtained after the interview: 9377 (78\%) participated from the target of 11971 invited (i.e. participants still in contact with the study and who at age 42 years had not required a proxy interview). Ethical approval for the age 45 year survey was given by the South East Multicentre Research Ethics Committee.

\section{Measures}

\section{Salivary cortisol}

Two saliva samples were collected in the morning, timed to capture the post-waking peak cortisol concentration and decline following this peak, as key characteristics of the normative cortisol diurnal rhythm. Accordingly, participants were asked to collect two saliva samples on the next convenient day after interview, the first $45 \mathrm{~min}$ after awaking (time 1 ) and the second $3 \mathrm{~h}$ later on the same day (time 2). Most participants $(n=9165)$ consented to saliva collection for cortisol measurement; a reminder was sent to $53 \%$ of those who consented but had not responded within 2 weeks of the nurse visit. Samples were received from 6568 participants, of whom 6524 had information on at least one cortisol measure (6467 for the time 1 measure, 6506 for time 2 and 6449 for both). The number responding is likely to reflect our reliance on participants to collect and return their samples.

Participants were instructed to avoid brushing or flossing their teeth and eating or drinking for $15 \mathrm{~min}$ before taking each sample. They were asked to chew on a cortisol collection swab (Salivette, Sarstedt, Numbrecht, Germany) until it was soaked, record the date and time of collection and store the sample at room temperature until posting it to the laboratory. Salivary cortisol is stable at room temperature for up to 30 days but samples were frozen after reaching the laboratory to reduce microbial growth. Cortisol levels were measured at the University of Dresden with a commercial chemiluminescence immunoassay kit (IBL International, Hamburg, Germany). The lower sensitivity of this assay is $0.44 \mathrm{nmol} / \mathrm{l}$, with intra-assay and interassay precision below $10 \%$ for a wide range of cortisol concentrations. Samples with cortisol levels above $50 \mathrm{nmol} / \mathrm{l}$ were rerun in a second assay for confirmation. Participants also reported whether they regularly worked at night (shift work); wakefulness during the previous night; dental work within the previous 3 days; cuts inside their mouth that might bleed; and current medications, categorised as a dichotomous variable. 


\section{Childhood psychosocial adversity}

Scales for maltreatment and household dysfunction were derived from information collected in childhood and adulthood. Maltreatment scales were constructed from several items selected to represent components of conventional definitions (neglect, abuse, witnessing abuse). ${ }^{6}$ Information on abuse (physical, sexual or psychological), neglect and witnessing physical or sexual abuse in others in the family was collected solely at age 45 years (see Appendix). A maltreatment scale was created by summing the scores on the three abuse items, one item on witnessing physical or sexual abuse in others in the family and three items on neglect (range 0-7). Further, from information collected in childhood we created an additional scale of neglect, by summing the eight items on the child's physical appearance and the parent's interaction with the child at ages 7 years, 11 years and 16 years (see Appendix). Information collected during childhood (at ages 7, 11 and 16 years) was obtained from structured questionnaires completed by the child's teacher and from a health visitor interview with parents (usually the mother). Information collected in adulthood (age 45 years) was obtained from participants who completed a confidential questionnaire about their childhood to age 16 years, using direct computer data entry. The questionnaire at age 45 years was derived from the Personality and Total Health (PATH) Through Life Project, ${ }^{10}$ originating from the Parental Bonding Instrument, the British National Survey of Health and Development and the US National Comorbidity Survey. Information on household dysfunction, collected in child and adulthood, was used to create a scale of 11 items. For items measured at more than one age, any positive response was classified as adversity. Data on separation or divorce collected from parent interviews at 11 years and 16 years of age were supplemented with data from cohort members at age 33 years.

\section{Confounding and mediating factors}

Socioeconomic position in childhood and adulthood and adult smoking were included because of their association with both cortisol levels ${ }^{11,12}$ and childhood adversity. ${ }^{6,13}$ Socioeconomic position at birth was based on the father's occupation using the UK Registrar General's social class categories, and grouped as professional/managerial, skilled non-manual, skilled manual and semi-skilled or unskilled manual, including single-mother households. Socioeconomic position at age 42 years was based on the participant's current or most recent occupation and categorised as above. Smoking habits, reported at 42 years, ranged from 'never' to 'current smoker, 20 cigarettes a day or more' (seven categories). Current psychological state at age 45 years was indicated by two or more symptoms of anxiety or depression on the nurse-administered revised Clinical Interview Schedule (CIS-R). ${ }^{14}$

\section{Statistical analysis}

Extreme cortisol outliers for time $1\left(T_{1}\right)$ and time $2\left(T_{2}\right)$ were truncated at $2 \mathrm{nmol} / \mathrm{l}$ for values below this level $\left(n=24\right.$ at $T_{1}$, $n=123$ at $T_{2}$ ) and at $100 \mathrm{nmol} / \mathrm{l}$ for values above $100 \mathrm{nmol} / \mathrm{l}$ ( $n=22$ at $T_{1}, n=20$ at $T_{2}$ ) in order that extreme values did not exert a disproportionate influence on analyses. Cortisol values were skewed, hence we transformed data using $\log 10\left(C_{1}\right.$ and $C_{2}$ ) to achieve a distribution approximating normality. Not all samples were collected at the specified periods after waking, leading to variation around the target time for $T_{1}$ (mean $49 \mathrm{~min}$, s.d. $=15 \mathrm{~min}$ ) and $T_{2}$ (mean $3 \mathrm{~h} 5 \mathrm{~min}$, s.d. $=23 \mathrm{~min}$ ). Given that cortisol level was influenced by both time of awaking and time since awaking, we centred the log-transformed cortisol values for each individual at $08.08 \mathrm{~h}(45 \mathrm{~min}$ after the mean awaking time of $07.23 \mathrm{~h})$ and $T_{2}$ at $11.08 \mathrm{~h}(3 \mathrm{~h} 45 \mathrm{~min}$ after mean awaking time) using coefficients for time of waking $\left(T_{0}\right), T_{1}$ and $T_{2}$ from linear regression models. Specifically, we fitted a linear regression model for $C_{1}$ on $T_{0}$ and $T_{1}: C_{1}=a+b T_{0}+c T_{1}$. For each individual the centred value for $C_{1}$ (Cort1) was derived as Cort $1=C_{1}+b\left(07.23 \mathrm{~h}-T_{0}\right)+c\left(08.08 \mathrm{~h}-T_{1}\right)$. Similarly, we derived centred cortisol values (at $11.08 \mathrm{~h}$ ) for $T_{2}$. Thus, $T_{1}$ and $T_{2}$ cortisol levels in all analyses were adjusted for both time of awaking and time since awaking.

To investigate associations with childhood adversity we analysed several cortisol measures derived from transformed and centred values: first, $T_{1}$ and $T_{2}$ cortisol levels; second, area under the curve (AUC), derived as the sum of $T_{1}$ and $T_{2}$ cortisol (Cort1 and Cort2 back-transformed to $\mathrm{nmol} / \mathrm{l}$, i.e. $10^{\text {Cort } 1}$ and $10^{\text {Cort2 }}$ ) multiplied by $3 \mathrm{~h}$ and divided by 2 (thus, AUC represents the $3 \mathrm{~h}$ average of $T_{1}$ and $T_{2}$ values, allowing for variation in collection times, used here to indicate total $3 \mathrm{~h}$ exposure); and third, cortisol $T_{1}$ to $T_{2}$ slope.

Continuous cortisol variables were analysed using linear regression. Given that $T_{1}$ and $T_{2}$ cortisol and AUC were $\log 10$ transformed, relative change (in percentages) in these cortisol measures was calculated from the regression coefficient $(\beta)$ as $100 \times\left(10^{\beta}-1\right)$. For example, $T_{1}$ cortisol changes from a value $x$ to $10^{\beta} x$ when the adversity increases by one level, e.g. from 0 to 1 on the maltreatment scale. Scales for adversity were modelled as continuous to identify trend across scores and also as categorical predictors to identify threshold associations. In initial analyses we examined potential influences on cortisol measurement: night work $(8 \%)$, awake during the previous night $(37 \%)$, cuts inside mouth $(3 \%)$, recent dental treatment $(2 \%)$, current medication and day of the week. Current medication was associated with higher $T_{1}$ and lower $T_{2}$ cortisol levels, whereas regular night work was associated with lower $T_{2}$ levels; hence, we adjusted for these factors in analyses. Because of gender differences in cortisol secretion, ${ }^{15}$ and in some childhood adversities, ${ }^{10}$ analyses were conducted separately by gender. However, gender differences were also tested by including an interaction term between gender and each adversity in combined models $(P<0.05)$. Four stages of regression models were undertaken:

(a) adjusted for factors affecting cortisol measurement (model 1);

(b) additionally adjusted for socioeconomic position in childhood and adulthood, and adult smoking (model 2);

(c) further adjusted for anxiety and depression symptoms at age 45 years (model 3 );

(d) with an interaction term between child maltreatment (or household dysfunction) score and current anxiety or depression to test effect modification.

Because of potential biases associated with sample attrition, ${ }^{16}$ non-response was handled by inverse probability weighting. Several factors associated with non-response at 45 years were used for the weighting, including gender, social class at birth, mathematics score at age 11 years and socioemotional behaviour at ages 7 years and 11 years. We repeated analyses using the sample with complete data and results were similar to those from weighted analyses; the latter are presented here. In addition, we checked the validity of our findings using sensitivity analyses in which assumptions varied about missing adversity measures obtained in adulthood. Specifically, we assumed that one, two or all missing data items were 'unexposed'. Results were similar across all analyses and conclusions were unaltered: here we present results assuming that individuals with up to two missing items were unexposed. 


\section{Results}

Childhood maltreatment and household dysfunction items and scores are shown for all participants at age 45 years briefly in Table 1 and in full in online Table DS1. The most common maltreatment items reported at this age were psychological abuse and absence of affection from the father. In terms of summary scores, $3.9 \%$ men and $5.4 \%$ women had two or more reports of abuse, whereas $1.7 \%$ and $3.1 \%$ respectively had two or more reports of neglect. On the overall maltreatment scale, 3.5\% men and $6.1 \%$ women had three or more maltreatments reported at this age. For the eight individual neglect items collected during childhood, many were common but only a minority ( $5.7 \%$ boys, $4.6 \%$ girls $)$ had five or more items. For household dysfunction the most prevalent items were maternal mental health problems and authoritarian upbringing. Even though some items were common, most individuals $(>80 \%)$ had two or fewer household dysfunction items; approximately $4 \%$ men and $7 \%$ women had five or more (Table 1). At age 45 years, $10.4 \%$ men and $14.6 \%$ women reported two or more depression or anxiety symptoms.

Table 2 presents information for those with at least one cortisol measure: men had a lower median cortisol level at $T_{1}$ but a higher level at $T_{2}(18.8 \mathrm{nmol} / 1$ and $7.1 \mathrm{nmol} / 1$ respectively $)$ than women $\left(19.6 \mathrm{nmol} / \mathrm{l}\right.$ and $6.6 \mathrm{nmol} / \mathrm{l}$ respectively). For $T_{1}$ cortisol there was no association with either abuse or household dysfunction in childhood or with depression/anxiety symptoms at 45 years (Table 3 ). In women but not in men, $T_{1}$ cortisol was

\begin{tabular}{|c|c|c|c|}
\hline & $n$ & $\begin{array}{l}\text { Men } \\
\%(n)\end{array}$ & $\begin{array}{l}\text { Women } \\
\%(n)\end{array}$ \\
\hline \multicolumn{4}{|l|}{ Childhood maltreatment reported at age 45 years $^{\mathrm{a}}$} \\
\hline Abuse & 9309 & & \\
\hline \multicolumn{4}{|l|}{ Number of types of abuse (0-3) } \\
\hline 1 & & $6.8(313)$ & $8.4(395)$ \\
\hline$\geqslant 2$ & & $3.9(181)$ & $5.4(255)$ \\
\hline Witnessed physical or sexual abuse of others in family & 9309 & $4.4(205)$ & $7.6(355)$ \\
\hline Neglect & 9308 & & \\
\hline \multicolumn{4}{|l|}{ Number of types of neglect (0-3) } \\
\hline 1 & & $10.3(474)$ & $10.0(467)$ \\
\hline$\geqslant 2$ & & $1.7(77)$ & $3.1(147)$ \\
\hline Maltreatment score (range $0-7$ ) & 9308 & & \\
\hline $1-2$ & & $15.6(721)$ & $16.4(769)$ \\
\hline $3-4$ & & 3.0 (137) & $4.8(224)$ \\
\hline$\geqslant 5$ & & $0.5(25)$ & $1.3(60)$ \\
\hline \multicolumn{4}{|l|}{ Childhood maltreatment reported during childhood ${ }^{a}$} \\
\hline Neglect $(7,11$ or 16 years) & 8324 & & \\
\hline \multicolumn{4}{|l|}{ Number of neglect items (range 0-8) } \\
\hline $1-2$ & & $37.3(1532)$ & $38.8(1636)$ \\
\hline $3-4$ & & $18.0(737)$ & $16.5(696)$ \\
\hline$\geqslant 5$ & & $5.7(234)$ & $4.6(192)$ \\
\hline \multicolumn{4}{|l|}{ Other psychosocial adversities } \\
\hline Household dysfunction score (range 0-11) ${ }^{a}$ & 9157 & & \\
\hline $1-2$ & & 42.5 (1931) & 39.9 (1841) \\
\hline $3-4$ & & $11.4(519)$ & $14.7(680)$ \\
\hline$\geqslant 5$ & & $4.0(180)$ & $6.7(308)$ \\
\hline \multicolumn{4}{|l|}{ Adult psychological state at 45 years } \\
\hline Depressive/anxiety symptoms (2 or more) & 9297 & $10.4(481)$ & $14.6(683)$ \\
\hline
\end{tabular}

\begin{tabular}{|c|c|c|c|c|}
\hline & \multicolumn{2}{|c|}{ Men } & \multicolumn{2}{|c|}{ Women } \\
\hline & & $n$ & & $n$ \\
\hline \multicolumn{5}{|l|}{ Time, h:min: mean (s.d.) } \\
\hline Waking time & $07.22(1: 22)$ & 3004 & $07.24(1: 12)$ & 3192 \\
\hline Time 1 & $08.12(1: 22)$ & 3159 & $08.13(1.12)$ & 3290 \\
\hline Time since waking & $0: 49(0: 15)$ & 3004 & $0: 49(0: 15)$ & 3189 \\
\hline Time 2 & $11.17(1: 26)$ & 3047 & $11.17(1: 13)$ & 3162 \\
\hline Interval (time 2 - time 1) & $3: 06(0: 26)$ & 3045 & 3:04 (0:18) & 3158 \\
\hline \multicolumn{5}{|l|}{ Cortisol measures, $\mathrm{nmol} / \mathrm{l}$} \\
\hline Time 1 cortisol: median ${ }^{a}$ & 18.80 & 3185 & $19.60^{* *}$ & 3282 \\
\hline Time 2 cortisol: median ${ }^{a}$ & 7.10 & 3198 & $6.60 * * *$ & 3308 \\
\hline Slope & -3.70 & 3174 & $-4.9^{* \star *}$ & 3275 \\
\hline AUC: median ${ }^{\mathrm{b}}$ & 40.11 & 3174 & 40.67 & 3275 \\
\hline \multicolumn{5}{|c|}{$\begin{array}{l}\text { AUC, area under the curve. } \\
\text { a. Truncated at } 2 \text { nmol/l and } 100 \mathrm{nmol} / \mathrm{l} \text {. } \\
\text { b. Calculated using centred time } 1 \text { and time } 2 \text { cortisol values, i.e. allowing for time of measurement. }\end{array}$} \\
\hline
\end{tabular}




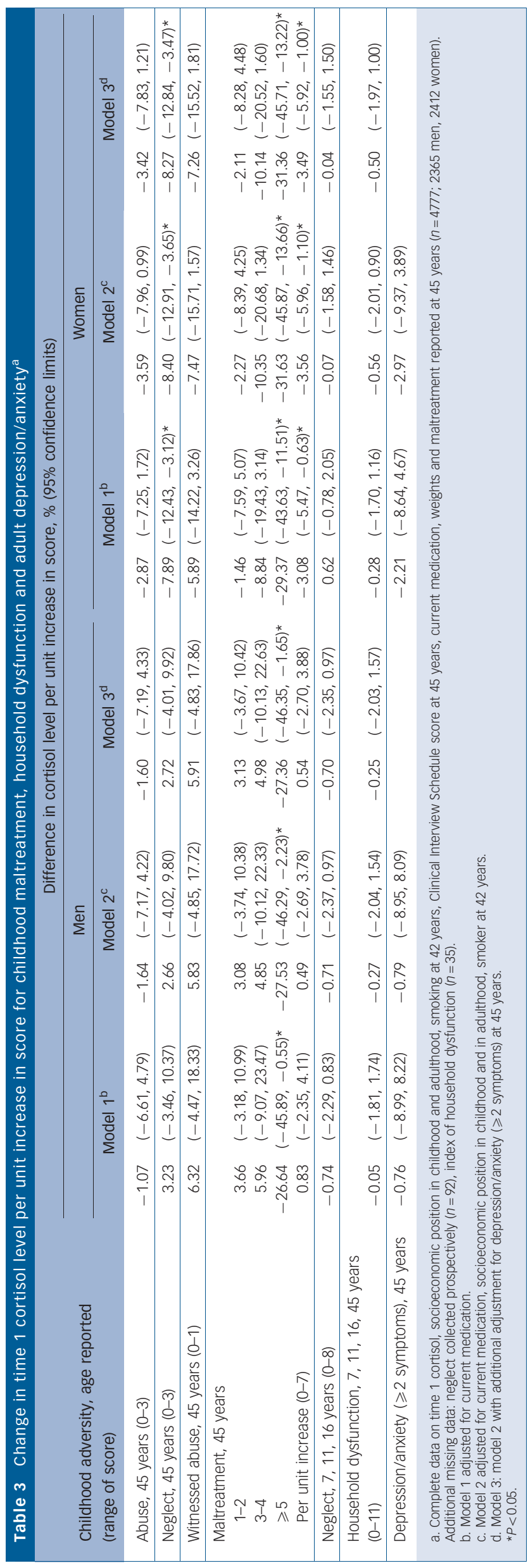

lowered by $7.9 \%$ per unit increase in childhood neglect score (range $0-3$ for 45 year score; gender interaction $P=0.01$ ). Also for women, there was a trend of lowered $T_{1}$ cortisol by $3.1 \%$ per unit increase in maltreatment score over the range $0-7$. No trend was seen for men, but for both men and women $T_{1}$ cortisol was lowered by more than $25 \%$ for those with five or more maltreatments $v$. no maltreatment, i.e. a dose-response relationship for women and threshold effect for men. All associations remained after adjustment for current medication, socioeconomic position in child and adulthood, adult smoking and depression/ anxiety. Among women the association between maltreatment score and $T_{1}$ cortisol level did not vary according to current depression or anxiety symptoms. In men the lower $T_{1}$ cortisol level for those with five or more maltreatments was seen only in those without (i.e. with fewer than two) depression or anxiety symptoms (for interaction $P=0.003$ ).

For $T_{2}$ cortisol there was no association with either childhood abuse or household dysfunction (Table 4). Men with a maltreatment score of 3 or more had an elevated $T_{2}$ cortisol (by approximately $30 \%$ ) compared with those with no maltreatment. This association remained although it was slightly attenuated after adjustment. A weaker (non-significant) trend with maltreatment score was observed for women. However, cortisol level at $T_{2}$ was elevated among women who witnessed abuse and there was a trend for neglect score (using childhood measures). These associations were weakened after adjustment partly owing to the elevated $T_{2}$ cortisol among women with current depression/ anxiety symptoms (Table 4 ).

Most participants had a higher cortisol levels at $T_{1}$ than at $T_{2}$, i.e. there was a negative $T_{1}$ to $T_{2}$ slope (Table 2). If $T_{1}$ is low and/ or $T_{2}$ is elevated the negative slope will be less steep, indicating a slower decline in cortisol levels over the morning. In women, neglect (age 45 years), witnessing abuse and maltreatment scores were associated with less steep negative slopes before and after adjustment (Table 5). Total $3 \mathrm{~h}$ cortisol exposure, indicated by AUC, was lower by $4.3 \%$ ( $95 \%$ CI -8.1 to -0.3 ) for each increment in neglect score (45 years) for women but not for men (gender interaction $P=0.01$ ). This association did not diminish after adjustment (data not presented).

\section{Discussion}

Cumulative maltreatments in childhood were associated with flattened morning cortisol secretion $\left(T_{1}\right.$ cortisol or $T_{1}$ to $T_{2}$ decline) in mid-adult life. For women, the total burden of childhood maltreatment was associated with reduced $T_{1}$ cortisol level at age 45 years, with a notable contribution of neglect (reported retrospectively) to this association. Specifically, $T_{1}$ cortisol declined by more than $3 \%$ for each increment across a 0-7 maltreatment scale, regardless of concurrent adult depressive and anxiety symptoms, and correspondingly there was a less steep morning $\left(T_{1}\right.$ to $\left.T_{2}\right)$ decline in cortisol levels. Consistently for both men and women, those with the most maltreatments (five or more) had lower $T_{1}$ cortisol levels by more than $25 \%$. In men, $T_{2}$ cortisol was strongly elevated for those with three or more maltreatments, even after adjustment; this pattern was similar, albeit weaker, for women. No independent association was found between childhood abuse or household dysfunction and $T_{1}$ or $T_{2}$ cortisol.

\section{Methodological considerations}

Ascertainment of childhood maltreatment and other psychosocial adversities is not straightforward because all ascertainment methods have biases and inconsistencies. ${ }^{6}$ Our neglect and 

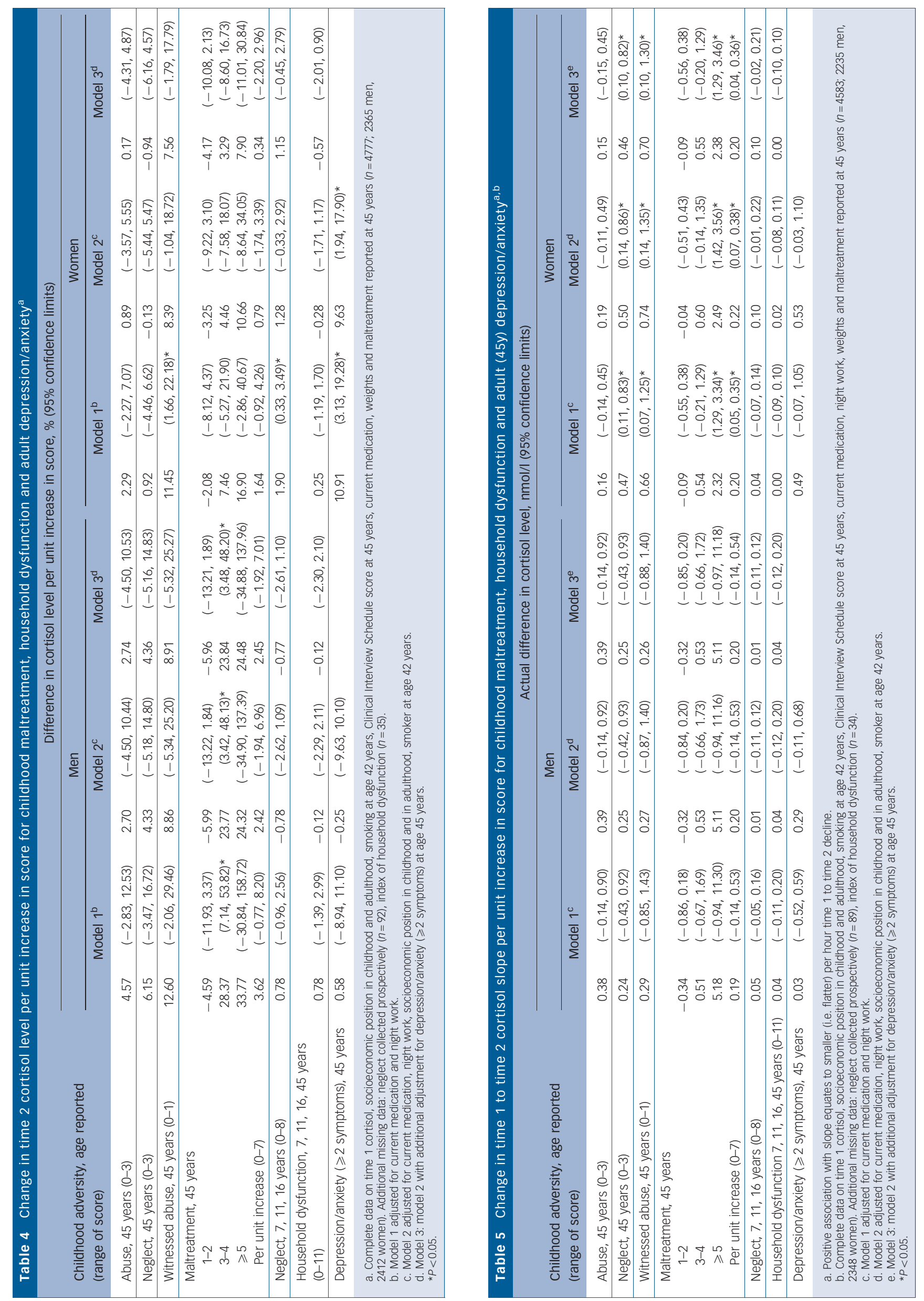
household dysfunction measures were based on information collected in childhood (from parents and teachers) and in adulthood from study participants. Measures of abuse were constructed from adult reports alone. There are different limitations associated with each method; information from parents may be influenced by socially desirable responding and concealment, whereas reports from study participants in adulthood may be affected by recollection. Nonetheless, we used conventional definitions for childhood abuse and neglect. For example, child neglect is defined as failure to meet a child's basic physical, emotional, medicinal/dental or educational need; to provide adequate nutrition, hygiene and shelter; or to ensure a child's safety. ${ }^{6}$ Our neglect measures collected in childhood largely - although not completely - capture this definition. Any single study, including ours, cannot entirely overcome inherent problems of measurement. However, we used multiple informants, time points and indicators to best identify a range of childhood adversities. Such a range of measures provides insights into whether associations with adult cortisol levels are robust to data ascertainment method. Wherever possible we created scores to reflect burden of adversity, rather than relying on any single item. It is also noteworthy that all reports of childhood adversity were collected masked to knowledge of cortisol levels.

A further consideration is our measurement of cortisol twice in the morning on one day. There is a lack of consensus on the measurement of HPA axis function; several measures are used, for example, based solely on cortisol or its ratio to dehydroepiandrosterone (DHEA) ${ }^{15}$ Cortisol measurement is usually timed to take account of the well-established normative diurnal rhythm of a post-waking peak followed by a decline over the next few hours. ${ }^{17}$ The cortisol awakening response (CAR) is frequently used, based on a measure immediately on waking and then at intervals (e.g. $30 \mathrm{~min}$ ) over the subsequent hour. ${ }^{17}$ Ideally, multiple saliva collections over several days best characterise an individual's diurnal cortisol rhythm, including measures such as CAR. We lacked a measure on waking so were unable to assess CAR, but our post-waking measure was timed to capture the period of peak concentration (a systematic review of CAR gives a range of $20-45 \mathrm{~min}$ after waking as the period of peak concentration, and several other studies have also used $45 \mathrm{~min}) .{ }^{17}$ In our large population study a maximum of two samples on one day was feasible. Our second cortisol measure was timed at $3 \mathrm{~h}$ after $T_{1}$ (i.e. before lunch) to assess whether levels declined as expected in accord with the normative diurnal rhythm. To summarise limitations, from our two morning measures we could not assess decline throughout the rest of the day or CAR, and no childhood cortisol measure was available. Nevertheless, the two samples provide an approximation of both post-waking peak concentration and the average rate of subsequent decline in adult cortisol level. Because of the study size, precision in estimation of effects is gained at the group level, although estimates for individuals may be less reliable. Accordingly, differences in adult cortisol patterns within this population have been shown for social classes and other groups. ${ }^{12}$ Furthermore, we found associations between current medication use and $T_{1}$ and $T_{2}$ cortisol. Given the numerous drugs and medical conditions that could affect cortisol, these associations warrant separate investigation. Owing to sample attrition complete data were available for less than half of the original cohort and underrepresented participants with the most adverse childhood backgrounds. We therefore undertook a weighted analysis using factors associated with non-participation to allow for differential loss to follow-up. ${ }^{16}$

Our study overcomes several limitations of research to date, such as clinical or special populations with specific types of adversity, ${ }^{18-20}$ small sample size, focus solely on women, ${ }^{2,18,21}$ or short-term follow-up. ${ }^{1,2}$ As it was a general population study we were able to capture experiences that vary widely in severity and may accumulate across types of adversity. If multiple adversities represent the most severely affected individuals, one could argue that population-based studies are less efficient than clinical studies. We argue that they are complementary because those with the greatest burden of adversity in a general population will not necessarily be identified through clinical and administrative referral.

\section{Interpretation of findings}

Childhood adversity, notably maltreatment, is associated with multiple health hazards many years later in adulthood. ${ }^{6}$ One potential biological mechanism linking childhood adversity and later outcome involves regulation of the HPA axis, although few studies exist to establish cortisol patterns into middle age. This is an important omission because the impact of childhood insults on cortisol regulation may evolve over the lifespan. Studies restricted to childhood have reported severe tactile deprivation in the first 2 years of life and sexual abuse of young girls to be associated with lower early morning cortisol levels, ${ }^{1,2}$ whereas diverse forms of maltreatment were associated with elevated levels. ${ }^{3}$ Some studies suggest that associations can persist into adulthood, such that severe childhood stress and trauma may be related to adult hypocortisolism. ${ }^{18,20-22}$ One notable prospective study highlighted the changing relationships with age: high cortisol levels were seen when childhood maltreatment was first experienced, followed by lower levels as the HPA axis evolved from child to adulthood differently in the maltreated and nonmaltreated groups. ${ }^{5}$ This pattern may be due to downregulation of the HPA system in the maltreated individuals in response to initially high levels of circulating glucocorticoids. ${ }^{23}$ Whether cortisol levels are elevated, reduced or show no differences in relation to child maltreatment may therefore depend on life stage, i.e. the time elapsed since maltreatment. Our findings need to be considered in light of such evolving life-course patterns, in that associations with cortisol in mid-adulthood might not be evident across all childhood maltreatments. Consistent with reports of adult hypocortisolism in relation to severe childhood stress and trauma, 5,18,20-22 we found incremental reductions in adult $T_{1}$ cortisol and flattened decline over the morning among women with accumulating burden of childhood maltreatment. Our study adds to the growing literature suggesting that the HPA axis may become downregulated in response to maltreatment in childhood, leading to a flattened cortisol diurnal rhythm. Cortisol pattern may have implications for subsequent health outcomes, with some recent studies suggesting that a flattened diurnal pattern is associated with poorer physical function at older ages, ${ }^{24}$ and increased risk of cardiovascular mortality. ${ }^{25}$

It has been argued that associations with cortisol patterns may differ, for example by type of maltreatment, ${ }^{22}$ possibly due to variation in age in childhood or developmental stage of the brain when different maltreatments occurred. ${ }^{4}$ In our study, variations were observed related to childhood adversity measures and gender. Although no association was observed for childhood abuse or household dysfunction, we found that women (but not men) had lower average $T_{1}$ cortisol with childhood neglect (at 45 years). These results parallel the findings from a study of mental health in which the risk of major depressive disorder was elevated in association with childhood neglect but not with sexual abuse. ${ }^{7}$ Women might be subject to more - or more upsetting - life events such as abuse than men, ${ }^{26}$ as observed in our study. Research also suggests that women are more vulnerable to effects of life events 
on mental health, ${ }^{27}$ possibly reflecting gender differences in social roles that enable men to distance themselves from life events. ${ }^{28}$ On the other hand, higher $T_{2}$ cortisol levels were seen in men but not in women in relation to cumulative maltreatment.

Our finding of lack of association between childhood neglect and adult cortisol level for men was robust to data ascertainment method, whereas for women associations varied for neglect ascertained in child and adulthood. Plausibly, current mental health could affect retrospective report of childhood adversities differently for men and women. Our analyses show higher $T_{2}$ cortisol for women with current symptoms of anxiety or depression, as suggested elsewhere. ${ }^{15}$ However, adjustment for current symptoms had little effect on the adversity-cortisol associations, for men or women. This does not support a 'reappraisal bias' according to current mental health. In the absence of population-based longitudinal data, the natural history of maltreatment and cortisol secretion remains a matter of speculation. Our observation of reduced adult $T_{1}$ cortisol with cumulative childhood maltreatment is consistent with reports of low cortisol output and psychiatric ill health in adulthood. ${ }^{29}$ However, our analyses suggest that altered adult cortisol secretion patterns associated with cumulative child maltreatments were not operating through current psychological state. Studies of samples such as the 1958 birth cohort have the capacity to put childhood maltreatment in the context of other influences that might mediate the association with adult cortisol secretion.

\footnotetext{
Chris Power, PhD, Medical Research Council (MRC) Centre of Epidemiology for Child Health/Centre for Paediatric Epidemiology and Biostatistics, University College London (UCL) Institute of Child Health, London; Claudia Thomas, PGCertHBE, Population (UCL) Institute of Child Health, London; Claudia Thomas, PGCertHBE, Population
Health Research Centre, Division of Population Health Sciences and Education, St George's University of London; Leah Li, PhD, MRC Centre of Epidemiology for Child Health/Centre for Paediatric Epidemiology and Biostatistics, UCL Institute of Child Health, London, UK; Clyde Hertzman, FRSC, Early Human Learning Partnership, University of British Columbia, Vancouver, Canada

Correspondence: Chris Power, UCL Institute of Child Health, 30 Guilford Street, London WC1N 1EH, UK. Email: christine.power@ucl.ac.uk

First received 3 May 2011, final revision 24 Apr 2012, accepted 14 May 2012
}

\section{Funding}

Analysis was funded by the UK Medical Research Council (MRC) (grant G0000934) and the Human Early Learning Partnership, Vancouver, Canada. The work was undertaken at the Great Ormond Street Hospital/University College London Institute of Child Health, London, who received a proportion of funding from the UK Department of Health's National Institute for Health Research Biomedical Research Centres funding scheme. The Centre for Paediatric Epidemiology and Biostatistics also benefits from funding support from the MRC in its capacity as the MRC Centre of Epidemiology for Child Health. L.L. was funded by an MRC career development award in biostatistics.

\section{Acknowledgements}

We are grateful to participants in the 2002-2004 clinical follow-up of the 1958 birth cohor and to the Medical Research Council for support of this survey. Cortisol levels were measured under the direction of Professor Kirschbaum, Biological Psychology, Department of Psychology, University of Dresden, Germany.

\section{Appendix}

\section{Questionnaire items at age $\mathbf{4 5}$ years}

Abuse

Psychological abuse by a parent (verbally abused or humiliated, ridiculed, bullied/mental cruelty)

Physical abuse by a parent (punched, kicked or hit or beaten with an object, or needed medical treatment)

sexual abuse by a parent

\section{Witnessed abuse}

Witnessed physical or sexual abuse of others in family

\section{Neglect}

Neglected

Father not at all affectionate

Mother not at all affectionate

\section{Childhood maltreatment reported during childhood}

Neglect (7, 11 or 16 years of age)

Scruffy, dirty or underfed appearance $(7,11$ years)

Mother hardly ever reads to child (7 years)

Father hardly ever reads to child (7 years)

Hardly ever takes outings with mother $(7,11$ years)

Hardly ever takes outings with father (7, 11 years)

Mother little interest in education (7, 11 or 16 years)

Father little interest in education (7, 11 or 16 years)

Low parental aspirations: leave school at minimum age (11 or 16 years)

\section{Other psychosocial adversities}

Household dysfunction $(7,11,16$ or 45 years)

Domestic tension ( 7,45 years)

Parental drink or drug problem (7, 45 years)

Doesn't get on well with mother (16 years)

Doesn't get on well with father (16 years)

Institutional care by age 16 years (16 years)

Father suffered from nervous or emotional trouble or depression (45 years)

Mother suffered from nervous or emotional trouble or depression (45 years)

Strict, authoritarian or regimented upbringing (45 years)

Too much physical punishment - hitting, smacking, etc. (45 years)

Separation or divorce of parents by age 16 years

Grew up in poverty (45 years)

\section{References}

1 Carlson M, Earls F. Psychological and neuroendocrinological sequelae of early social deprivation in institutionalized children in Romania. Ann $N$ Y Acad Sci 1997: 807: 419-28.

2 King JA, Mandansky D, King S, Fletcher KE, Brewer J. Early sexual abuse and low cortisol. Psychiatry Clin Neurosci 2001; 55: 71-4.

3 Cicchetti D, Rogosch FA. Diverse patterns of neuroendocrine activity in maltreated children. Dev Psychopathol 2001; 13: 677-93.

4 Lupien SJ, McEwen BS, Gunnar MR, Heim C. Effects of stress throughout the lifespan on the brain, behaviour and cognition. Nat Rev Neurosci 2009; 10 : 434-45.

5 Trickett PK, Noll JG, Susman EJ, Shenk CE, Putnam FW. Attenuation of cortisol across development for victims of sexual abuse. Dev Psychopathol 2010; 22: 165-75.

6 Gilbert R, Widom CS, Browne K, Fergusson D, Webb E, Janson S. Burden and consequences of child maltreatment in high-income countries. Lancet 2009; 373: $68-81$.

7 Horwitz AV, Widom CS, McLaughlin J, White HR. The impact of childhood abuse and neglect on adult mental health: a prospective study. $J$ Health SOC Behav 2001; 42: 184-201.

8 Heim $C$, Nemeroff $C B$. The role of childhood trauma in the neurobiology of mood and anxiety disorders: preclinical and clinical studies. Biol Psychiatry 2001; 49: 1023-39.

9 Power C, Elliott J. Cohort profile: 1958 British birth cohort (National Child Development Study). Int J Epidemiol 2006; 35: 34-41.

10 Rosenman S, Rodgers B. Childhood adversity in an Australian population. Soc Psychiatry Psychiatr Epidemiol 2004; 39: 695-702.

11 Badrick E, Kirschbaum C, Kumari M. The relationship between smoking status and cortisol secretion. J Clin Endocrinol Metab 2007; 92: 819-24.

12 Li L, Power C, Kelly S, Kirschbaum C, Hertzman C. Life-time socio-economic position and cortisol patterns in mid-life. Psychoneuroendocrinology 2007; 32: $824-33$ 
13 Anda RF, Croft JB, Felitti VJ, Nordenberg D, Giles WH, Williamson DF, et al. Adverse childhood experiences and smoking during adolescence and adulthood. JAMA 1999; 282: 1652-8.

14 Lewis G, Pelosi AJ, Araya R, Dunn G. Measuring psychiatric disorder in the community: a standardized assessment for use by lay interviewers. Psycho Med 1992; 22: 465-86

15 Herbert J, Goodyer IM, Grossman AB, Hastings MH, De Kloet ER, Lightman SL, et al. Do corticosteroids damage the brain? J Neuroendocrinol 2006; 18: 393-411.

16 Atherton K, Fuller E, Shepherd P, Strachan DP, Power C. Loss and representativeness in a biomedical survey at age 45 years: 1958 British birth cohort. J Epidemiol Community Health 2008; 62: 216-23.

17 Chida $Y$, Steptoe A. Cortisol awakening response and psychosocial factors: a systematic review and meta-analysis. Biol Psychol 2009; 80: 265-78.

18 Roy A. Urinary free cortisol and childhood trauma in cocaine dependent adults. J Psychiatr Res 2002; 36: 173-7.

19 Luecken LJ, Appelhans BM. Early parental loss and salivary cortisol in young adulthood: the moderating role of family environment. Dev Psychopathol 2006; 18: 295-308

20 Weissbecker I, Floyd A, Dedert E, Salmon P, Sephton S. Childhood trauma and diurnal cortisol disruption in fibromyalgia syndrome. Psychoneuroendocrinology 2006; 31: 312-24.

21 Stein MB, Yehuda R, Koverola C, Hanna C. Enhanced dexamethasone suppression of plasma cortisol in adult women traumatized by childhood sexual abuse. Biol Psychiatry 1997; 42: 680-6.
22 Gunnar MR, Vasquez DM. Low cortisol and a flattening of expected daytime rhythm: potential indices of risk in human development. Dev Psychopathol 2001: 13: 515-38.

23 Dozier $\mathrm{M}$, Peloso $\mathrm{E}$. The role of early stressors in child health and mental health outcomes. Arch Pediatr Adolesc Med 2006; 160: 1300-1.

24 Gardner MP, Lightman SL, Gallacher J, Hardy R, Kuh D, Ebrahim S, et al. Diurnal cortisol patterns are associated with physical performance in the Caerphilly Prospective Study. Int J Epidemiol 2011; 40: 1693-702.

25 Kumari M, Shipley M, Stafford M, Kivimaki M. Association of diurnal patterns in salivary cortisol with all-cause and cardiovascular mortality: findings from the Whitehall II Study. J Clin Endocrin Metab 2011; 96: 1478-85.

26 Bebbington PE. Sex and depression. Psychol Med 1998; 28: 1-8.

27 van Os J, Jones P, Lewis G, Wadsworth M, Murray R. Developmental precursors of affective illness in a general population birth cohort. Arch Gen Psychiatry 1997; 54: 625-31.

28 Nazroo JY, Edwards AC, Brown GW. Gender differences in the onset of depression following a shared life event: a study of couples. Psychol Med 1997; 27: 9-19.

29 Strickland PL, Deakin JFW, Percival C, Dixon J, Gater RA, Goldberg DP. Bio-social origins of depression in the community. Interactions between social adversity, cortisol and serotonin neurotransmission. $\mathrm{Br} J$ Psychiatry 2002: 180: 168-73.

EXTRA

\title{
poem
}

\section{So This Is Death}

\author{
For Naomi, 1966-2003
}

\section{Bear Loveday Tyler}

So this is death. The silent thief Who came and stole our last goodbyes Now slowly turns the wheels of grief.

My golden girl. No lover and no friend Could blunt those bitter thorns in-turned Which overcame you, shaped the end

Which shocks us all. With one last sigh You turned your face to life's wild chief To end your pain. Your tears are dry

And fall no longer. You'd well earned Your final sunshine, gold to spend on your long journey, as you turned. Death rips a wound no tears can mend.

This poem is from Bear Loveday Tyler's book Love Grenade, published by Survivors' Poetry in 2006. Loveday was mentored by Robin Ford.

Chosen by Femi Oyebode. 